\title{
CONVEX-CYCLIC MATRICES, CONVEX-POLYNOMIAL INTERPOLATION AND INVARIANT CONVEX SETS
}

\author{
Nathan S. Feldman And Paul McGuire
}

Abstract. We define a convex-polynomial to be one that is a convex combination of the monomials $\left\{1, z, z^{2}, \ldots\right\}$. This paper explores the intimate connection between peaking convexpolynomials, interpolating convex-polynomials, invariant convex sets, and the dynamics of matrices. In particular, we use these intertwined relations to both prove which matrices are convexcyclic while at the same time proving that we can prescribe the values and a finite number of the derivatives of a convex-polynomial subject to certain natural constraints. These properties are also equivalent to determining those matrices whose nonempty invariant closed convex sets are all invariant subspaces.

Our characterization of the convex-cyclic matrices gives a new and correct proof of a similar result by Rezaei that was stated and proven incorrectly.

Mathematics subject classification (2010): 47A16, 15A60.

Keywords and phrases: Cyclic, convex-cyclic, orbit, convex-polynomial, polynomial interpolation.

\section{REFERENCES}

[1] A. Ayadi, H. Marzougui, Abelian semigroups of matrices on $\mathbb{C}^{n}$ and hypercyclicity, Proc. Edinb. Math. Soc. (2) 57 (2014), no. 2, 323-338.

[2] F. Bayart, E. Matheron, Dynamics of linear operators, Cambridge Tracts in Mathematics, 179, Cambridge University Press, Cambridge, 2009.

[3] T. Bermúdez, A. Bonilla, \& N. S. Feldman, On convex-cyclic operators, J. Math. Analysis \& Appl. 434, no. 2, (2016) p. 1166-1181.

[4] J. B. Conway, A Course in Functional Analysis, 2nd Edition, Springer-Verlag, 1990.

[5] G. Costakis, D. Hadjiloucas, A. Manoussos, Dynamics of tuples of matrices, Proc. Amer. Math. Soc. 137 (2009), no. 3, 1025-1034.

[6] G. Costakis And I. Parissis, Dynamics of tuples of matrices in Jordan form, Oper. Matrices 7 (2013), no. 1, 131-157.

[7] L. Elsner, On matrices leaving invariant a nontrivial convex set, Linear Algebra Appl. 42 (1982), 103-107.

[8] K. Grosse-Erdmann, A. Peris, Linear Chaos, Universitext. Springer, London, 2011.

[9] I. GohberG, P. LANCASTER, AND L. RodmAn, Invariant Subspaces of Matrices with Applications, Canadian Mathematical Society, John Wiley \& Sons, 1986.

[10] R. A. Horn And C. R. Johnson, Matrix Analysis, Cambridge University Press, 1999.

[11] F. LeÓn-S Aavedra, M. P. Romero-De LA Ros A, Powers of convex-cyclic operators, Abstract and Applied Analysis, vol. 2014 (2014), Article ID 631894, 3 pages.

[12] H. REZAEI, On the convex hull generated by orbit of operators, Linear Algebra Appl., 438 (2013), 4190-4203. 